\title{
Atuação do produto nanotecnológico à base de substâncias orgânicas sobre as perdas no arranquio do amendoim
}

\section{http://dx.doi.org/10.17648/sas.v111.93}

Samira Luns Hatum de Almeida (D)

Doutoranda em Agronomia (Produção Vegetal) - Universidade Estadual Paulista "Júlio de Mesquita Filho", samira.Ih.almeida@unesp.

Jarlyson Brunno Costa Souza iD

Mestrando em Agronomia (Produção Vegetal) - Universidade Estadual Paulista "Júlio de Mesquita Filho", jarlyson.brunno@unesp.br.

Pós-doutoranta em Agronomia (Produção Vegetal) - Universidade Estadual Paulista "Júlio de Mesquita Filho", franmorlin1@gmail.com.

Armando Lopes Brito (D)

Mestrando em Agronomia (Ciência do Solo) - Universidade Estadual Paulista "Júlio de Mesquita Filho", armando.brito@unesp.br.

Cristiano Zerbato (D)

Professor Dr. - Universidade Estadual Paulista “Júlio de Mesquita Filho”, cristiano.zerbato@unesp.br.

\section{RESUMO}

A cultura do amendoim enfrenta inúmeros obstáculos durante o seu cultivo que podem prejudicar significativamente a produção e o retorno econômico da atividade. Diante disso, objetivou-se com este trabalho avaliar os impactos de produto à base de substâncias orgânicas sobre produtividade e perdas decorrentes do arranquio na cultura do amendoim. Para isso, foi realizado experimento em área agrícola de Ribeirão Preto - SP. $O$ experimento possuiu delineamento em faixa contendo quatro tratamentos, um tratamento controle (testemunha) e três tratamentos com doses de produto nanotecnológico à base de complexo de substâncias orgânicas para a cultura do amendoim, e seis repetições, perfazendo 24 parcelas. Este produto foi aplicado aos 30 e 60 DAS (após a semeadura). As variáveis analisadas foram maturação, perdas e produtividade. A maturação foi avaliada aos 120 DAS e quanto as demais foram feitas no dia do arranquio. $O$ tratamento que continha aplicação do produto somente na semeadura e aos 30 DAS foi o que apresentou maior produtividade bem como vagens maduras. Em relação às perdas, o maior valor foi observado no tratamento controle. Foi verificado que a aplicação do produto proporcionou maiores quantidade de vagens maduras, e o aumento da produtividade.

Palavras-chave: Arachis hypogaea L.; Colheita mecanizada; Maturação.

\section{Performance of the nanotechnological product based on organic substances on losses in peanut digger}

\begin{abstract}
The peanut crop faces numerous obstacles during their growth that can significantly harm the production and the economic return of the activity. Therefore, the objective of this work was to evaluate the impacts of product based on organic substances on yield and losses, from peanut diggers. The study was conducted in an agricultural area located in the municipality of Ribeirão Preto/SP. The experiment had a strip design containing four treatments, a control treatment (control) and three treatments with doses of nanotechnological product based on a complex of organic substances for the peanut crop, and six replications, in totally 24 plots. This product was applied at 30 and 60 DAS (after sowing). The variables analyzed were maturation, losses,
\end{abstract}


and yield. The maturation was evaluated at 120 DAS and how much the others variables were made on the day of the digger. The treatment that contained the application of the product only at sowing and at 30 DAS was the one that showed the highest yield as well as mature pods. Regarding losses, the highest value was observed in the control treatment. It was found that the application of the product provided a greater amount of ripe pods and increased yield.

Keywords: Arachis hypogaea L.; Mechanized harvest; Maturation.

\title{
Rendimiento del producto nanotecnológico basado en sustancias orgánicas en pérdidas en el desarraigo de maní
}

\author{
RESUMEN
}

El cultivo de maní enfrenta numerosos obstáculos durante su cultivo que pueden dañar significativamente la producción y el retorno económico de la actividad. Por lo tanto, el objetivo de este trabajo fue evaluar los impactos del producto basado en sustancias orgánicas en la productividad y las pérdidas resultantes del desarraigo en el cultivo de maní. Para ello, se realizó un experimento en una zona agrícola de Ribeirão Preto - SP. El experimento tuvo un diseño de tira que contenía cuatro tratamientos, un tratamiento de control (control) y tres tratamientos con dosis de producto nanotecnológico basado en un complejo de sustancias orgánicas para el cultivo de maní, y seis repeticiones, con un total de 24 parcelas. Este producto se aplicó a 30 y 60 DAS (después de la siembra). Las variables analizadas fueron maduración, pérdidas y productividad. La maduración se evaluó a 120 DAS y cuánto se hicieron los demás el día del retiro. El tratamiento que contenía la aplicación del producto solo en la siembra y a los 30 DAS fue el que mostró la mayor productividad y las vainas maduras. En cuanto a las pérdidas, el valor más alto se observó en el tratamiento de control. Se descubrió que la aplicación del producto proporcionaba una mayor cantidad de vainas maduras $y$ aumentaba la productividad.

Palabras clave: Arachis hypogaea L., cosecha mecanizada, maduración, productividad.

\section{Introdução}

Uma das leguminosas de maior importância agrícola, o amendoim, tem sido amplamente cultivada em decorrência de sua alta adaptabilidade a diferentes condições edafoclimáticas e as diversas formas de consumo deste grão (CAVICHIOLI et al., 2014). No Brasil, o cultivo do amendoim tem papel fundamental no processo de rotação de culturas, sendo amplamente empregado na sucessão da cana-de-açúcar em áreas de reforma de canavial (ORMOND et al., 2018).

Mesmo com a valorização frequente do produto, a cultura do amendoim enfrenta inúmeros obstáculos durante o seu cultivo que podem prejudicar significativamente o retorno econômico da atividade. Um dos principais gargalos da cultura ocorre durante a operação de arranquio, primeira etapa da colheita. Por se desenvolver sob o solo, vagens de amendoim podem ficar retidas sob o solo durante esta operação (perdas 
invisíveis) contribuindo com as perdas decorrentes da colheita mecanizada. Entre os fatores que podem estar relacionados as perdas, pode-se citar, o clima, sanidade da lavoura, maturação das vagens, regulação de máquinas, e condições do solo (ZERBATO et al., 2017).

Uma das formas de aumentar a produtividade e reduzir as perdas decorrentes da colheita mecanizada na cultura do amendoim é por meio de produtos que contribuam para melhorar o desenvolvimento da planta. Neste contexto, destaca-se um produto produzido por meio de nanotecnologia à base de complexo de substâncias orgânicas capaz de nutrir e estimular o crescimento de microrganismos benéficos para as plantas.

Diante disso, objetivou-se com este trabalho avaliar os impactos de produto à base de substâncias orgânicas sobre produtividade e perdas decorrentes do arranquio na cultura do amendoim.

\section{Material e métodos}

O experimento foi realizado em área agrícola localizada no município de Ribeirão Preto - SP. A área, de solo Latossolo Vermelho, é de reforma de canavial e se encontrava em sistema de meiosi (Método Inter-rotacional Ocorrendo Simultaneamente) com cana-de-açúcar.

O amendoim semeado foi da cultivar IAC OL3, safra 2018/2019. Para a semeadura utilizou-se trator da marca John Deere, modelo 7195J (195 cv) com semeadora da marca Jumil de 6 linhas espaçadas em 0,9 m. Junto a este conjunto, foi acoplado máquina de injeção no sulco de semeadura para aplicação do produto à base de substâncias orgânicas.

O experimento consistiu na avaliação de doses do produto nanotecnológico à base de complexo de substâncias orgânicas para a cultura do amendoim e foi conduzido em faixas com quatro tratamentos e 6 repetições, totalizando 24 parcelas. Os tratamentos avaliados foram: T1 Tratamento controle (testemunha); $\mathrm{T} 2$ - $3 \mathrm{~g} \mathrm{ha}^{-1}$ (semeadura) $+5 \mathrm{~g} \mathrm{ha}^{-1}$ (30 DAS); T3 - $3 \mathrm{~g} \mathrm{ha}^{-1}$ (semeadura) + $5 \mathrm{~g} \mathrm{ha}^{-1}$ (30 DAS) $+5 \mathrm{~g} \mathrm{ha}^{-1}$ (60 DAS); T4 
- $5 \mathrm{~g} \mathrm{ha}^{-1}$ (semeadura) $+7,5 \mathrm{~g} \mathrm{ha}^{-1}$ (30 DAS) $+7,5 \mathrm{~g} \mathrm{ha}^{-1}$ (60 DAS) - Figura 1.



Figura 1. Arranjo experimental. Tratamentos entre linhas de cana-de-açúcar.

Para as aplicações aos 30 e 60 dias após a semeadura (DAS) utilizou-se o pulverizador costal a $\mathrm{CO}_{2}$. Cada grama do produto foi diluído em $100 \mathrm{~mL}$ de água. Nos dois mecanismos de aplicação, empregou-se volume de calda de $100 \mathrm{~L} \mathrm{ha}^{-1}$. A análise de maturação foi realizada com base no método Peanut Maturity Index (PMI), que calcula a porcentagem de vagens maduras para cada amostra. Para isso, amostras coletadas aos 120 DAS tiveram todas as vagens de amendoim destacadas das plantas. A maturação de amendoim é avaliada com base no método Hull-Scrape e no quadro de maturação (WILLIAMS \& DREXLER, 1981) (Figura 2).

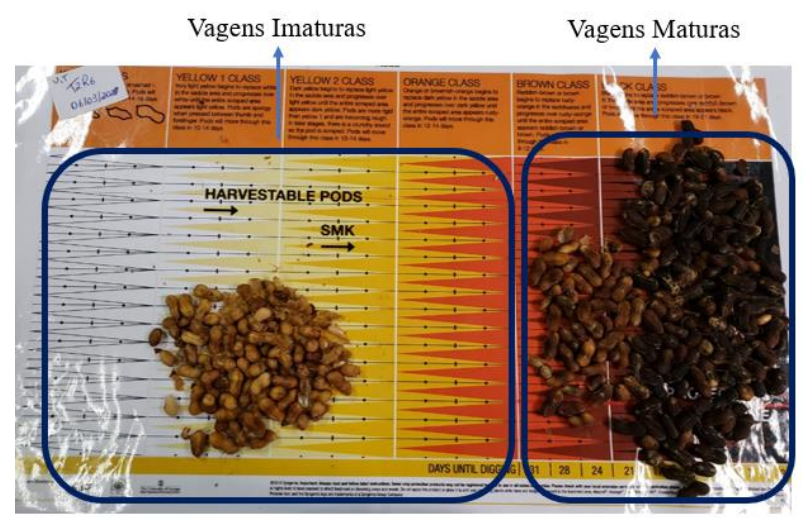

Figura 2. Maturação de amendoim avaliada com base no método HullScrape e no quadro de maturação (WILLIAMS \& DREXLER, 1981). 
A colheita do experimento foi iniciada no dia 06 de março de 2020. O trator empregado nesta operação foi o John Deere 7195J (195 cv) com arrancador-invertedor KBM 6×3 (arranca 6 linhas e faz 3 leiras). Após 0 arranquio foi realizada a coleta da produtividade e perdas provenientes da operação. Para as perdas utilizou-se armação retangular de 5,4 x 0,37 m, de área total de $2 \mathrm{~m}^{2}$, sendo está disposta sobre três linhas de amendoim. As perdas visíveis consistiram na coleta das vagens sobre o solo dentro desta armação. As perdas invisíveis, obtidas com auxílio da enxada, foram coletadas após o revolvimento do solo até a profundidade de $15 \mathrm{~cm}$. As perdas totais da operação foram calculadas a partir do somatório das perdas visíveis e invisíveis.

A produtividade foi coletada com uma armação de $2 \times 1 \mathrm{~m}$. Toda as plantas contidas nesta área tiveram as vagens destacadas das plantas. As vagens oriundas das amostras de produtividade e perdas foram pesadas em balança analítica e levadas a estufa a $75^{\circ} \mathrm{C}$ por $72 \mathrm{~h}$. Em seguida, o teor de água de ambas as variáveis foram corrigidas para $8 \%$ e os valores aferidos foram extrapolados para $\mathrm{kg} \mathrm{ha}^{-1}$. As variáveis foram submetidas a análise de variância e teste t de Student a 5\% de probabilidade.

\section{Resultados e discussão}

Os resultados de perdas visíveis, invisíveis, totais e a produtividade para os tratamentos analisados encontram-se na Figura 3. As análises estatísticas foram realizadas entre as colunas de mesma cor para os tratamentos. As produtividades observadas nos quatro tratamentos se diferiram em $1722,03 \mathrm{~kg} / \mathrm{ha}$. O T3 foi aquele que apresentou menor produtividade, seguido do T4 com $6959,85 \mathrm{~kg} / \mathrm{ha}$ e do T1 com 8088,85 $\mathrm{kg} / \mathrm{ha}$. O T3 diferiu-se estatisticamente somente do tratamento T2, de maior produtividade com $8253,47 \mathrm{~kg} / \mathrm{ha}$.

A maior produtividade foi atingida pelo tratamento que recebeu a menor quantidade de produto orgânico (T2). Tal comportamento pode indicar que a dose em questão, $3 \mathrm{~g} \mathrm{ha}^{-1}$ na semeadura $+5 \mathrm{~g} \mathrm{ha}^{-1}$ aos 30 DAS, está próxima ao ideal e que acréscimos na dose não acarretam incremento de 
produtividade que justifiquem seu uso. Além disso, observa-se que os tratamentos que tiveram aplicação aos 60 DAS foram os que apresentaram menores produtividades, o que leva a inferir que o produto gera melhores resultados para a planta quando aplicado no início do ciclo.

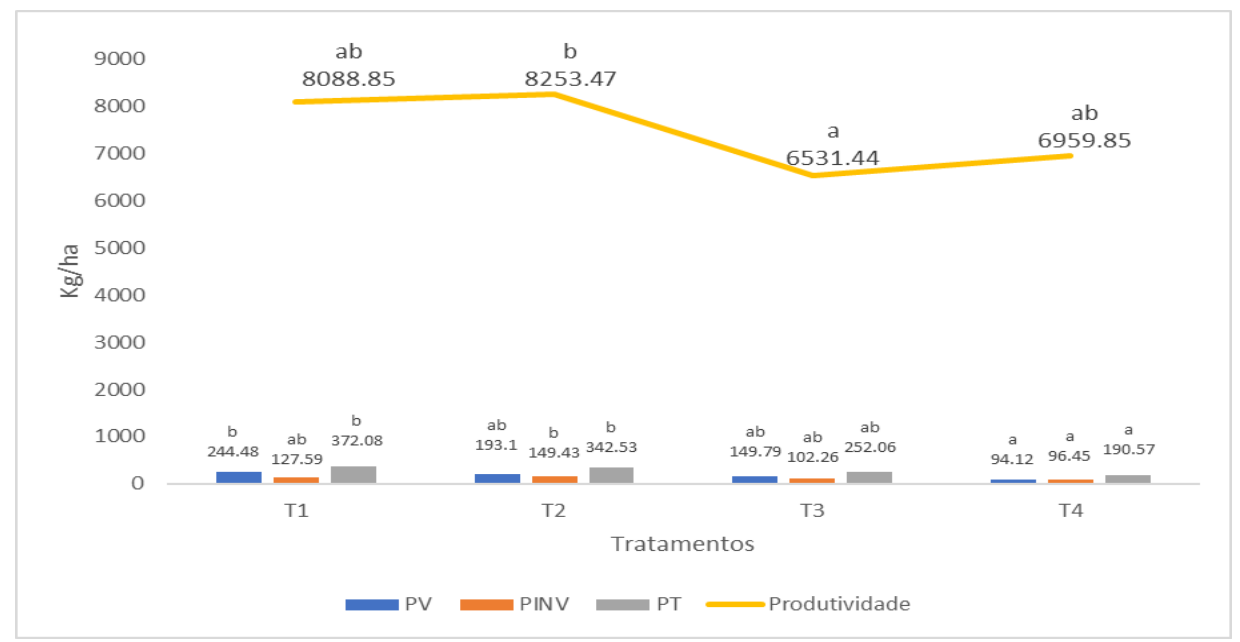

Figura 3. Dados de perdas e produtividade. $P V=$ perdas visíveis; $P I N V=$ perdas invisíveis; $\mathrm{PT}=$ perdas totais.

Ao analisar as perdas visíveis (coluna azul) pode-se observar que 0 T4 foi o que apresentou o menor valor em $\mathrm{kg} \mathrm{ha}^{-1}$, seguido do T3, T2 e T1. O T4, com perdas visíveis de $96,45 \mathrm{~kg} / \mathrm{ha}$ foi estatisticamente igual aos tratamentos T3 e T2, e diferiu-se somente do tratamento com maiores perdas, o tratamento controle, sem aplicação do produto (T1).

Para as perdas invisíveis (coluna laranja), as maiores perdas foram nos tratamentos $\mathrm{T} 2$ e $\mathrm{T} 1$. $\mathrm{O}$ tratamento $\mathrm{T} 2$ não se diferiu dos tratamentos $\mathrm{T} 1$ e T3 para a variável em questão. Um dos fatores que pode estar associado as altas perdas invisíveis no T2 é a maior produtividade entre os tratamentos. Em porcentagem, o tratamento em questão obteve $1,78 \%$ de perdas invisíveis, frente a $1,55 \%$ de perdas no $\mathrm{T} 1$, com a segunda maior produtividade. Além disso, o tratamento T2 foi o que apresentou a segunda maior porcentagem de vagens maduras, condição que favorece a deterioração do pedúnculo e consequentemente que estas se soltem da planta na operação de arranquio (ROBERSON, 2009) (Tabela 1).

Nas perdas totais (coluna cinza), o T4 foi o que apresentou menores valores de perdas, e o $\mathrm{T} 1$, aquele que apresentou maiores valores de perdas 
totais. Ao avaliar a porcentagem de perdas diante da produtividade, o tratamento controle, T1, apresentou maiores perdas totais, 4,39\%. Os demais tratamentos obtiveram perdas inferiores a $4 \%$, tendo os tratamentos T2 e T3 perdas de 3,98\% e 3,71\%, respectivamente. A menor porcentagem de perdas foi observada no tratamento $\mathrm{T} 4$, com $2,66 \%$. Os menores valores de perdas observados nos tratamentos com aplicação do produto orgânico podem estar associados à possível fortalecimento da planta e do pedúnculo. Isso porque, o produto em questão, à base de complexo de substâncias orgânicas, é capaz de promover o crescimento de microrganismos presentes nos solos e nas plantas, de modo a beneficiar o desenvolvimento da cultura.

$\mathrm{Na}$ Tabela 1 estão descritas as porcentagens de vagens imaturas e maduras. Ao analisar as porcentagens de frutos maduros nos tratamentos, pode-se observar que a maior porcentagem de frutos maduros ocorreu nos três tratamentos com aplicação do produto. $O$ tratamento controle foi o que apresentou menor porcentagem de frutos maduros. Com base nisso, o produto pode ter sido responsável por acelerar a maturação das vagens de amendoim. Esta é uma condição favorável, uma vez que ao atingir a maturação com menor espaço de tempo, a operação de arranquio pode ser iniciada antes, de modo a permitir liberação da área para o plantio de canade-açúcar.

Tabela 1. Análise de estágio de maturação das vagens.

\begin{tabular}{ccc}
\hline Tratamentos & Vagens imaturas (\%) & Vagens maduras (\%) \\
\hline T1 & 30,55 & 69,45 \\
T2 & 25,84 & 74,16 \\
T3 & 25,47 & 74,53 \\
T4 & 28,16 & 71,84 \\
\hline
\end{tabular}

\section{Conclusões}

O tratamento T2, com a utilização do produto à base de substâncias orgânicas na semeadura e aos 30 DAS, foi o que apresentou maior produtividade. As maiores perdas totais decorrentes da operação de arranquio foram observadas no tratamento sem aplicação do produto. 


\section{Referências}

CAVICHIOLI, F. A., ZERBATO, C., BERTONHA, R. S., \& DA SILVA, R. P. Perdas quantitativas de amendoim nos períodos do dia em sistemas mecanizados de colheita. Científica, n. 42, v.3, p. 211-215, 2014. http://dx.doi.org/10.15361/1984-5529.2014v42n3p211-215

ORMOND, A. T., DOS SANTOS, A. F., ALCANTARA, A. S., ZERBATO, C., \& FURLANI, C. E. Tillage interference in the quality of peanut mechanized harvest. Engenharia Agrícola, v. 38, v.2, p. 251-259, 2018. http://dx.doi.org/10.1590/1809-4430-eng.agric.v38n2p251-259/2018

ZERBATO C., FURLANI C. E. A, SILVA R. P., VOLTARELLI M. A., SANTOS A. F. Controle estatístico de processos aplicados para escavação mecânica de amendoim em classes de textura do solo. Engenharia Agrícola n. 37, v.2, p. 315-322, 2017. DOI: http://dx.doi.org/10.1590/1809-4430eng.agric.v37n2p315-322/2017

ROBERSON, G. T. Planting, harvesting, and curing peanuts. p.131-148. In: JORDAN, D. L. et al.; Peanut information 2010. North Carolina Coop. Ext. Ser. Series AG-331. 2009.

WILLIAMS, E.J., DREXLER, J. S.; A non-destructive method for determining peanut pod maturity. Peanut Science, v. 8, n. 2, p. 134-141, 1981. 